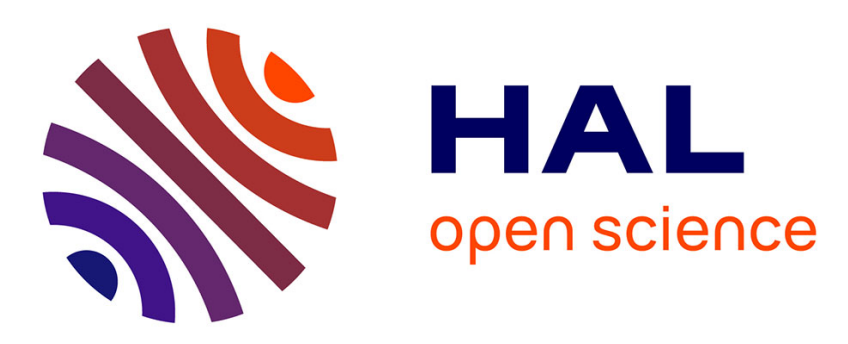

\title{
Stability Analysis for Neutral and Time-Varying Systems Using Linear Lyapunov Functionals and a Positive Systems Approach
}

Frédéric Mazenc, Michael Malisoff

\section{- To cite this version:}

Frédéric Mazenc, Michael Malisoff. Stability Analysis for Neutral and Time-Varying Systems Using Linear Lyapunov Functionals and a Positive Systems Approach. ASME 2014 Dynamic Systems and Control Conference, Oct 2014, San Antonio, United States. 10.1115/DSCC2014-6218 . hal-01088236

\section{HAL Id: hal-01088236 \\ https://inria.hal.science/hal-01088236}

Submitted on 24 Dec 2014

HAL is a multi-disciplinary open access archive for the deposit and dissemination of scientific research documents, whether they are published or not. The documents may come from teaching and research institutions in France or abroad, or from public or private research centers.
L'archive ouverte pluridisciplinaire HAL, est destinée au dépôt et à la diffusion de documents scientifiques de niveau recherche, publiés ou non, émanant des établissements d'enseignement et de recherche français ou étrangers, des laboratoires publics ou privés. 
DSCC2014-6218

\section{STABILITY ANALYSIS FOR NEUTRAL AND TIME-VARYING SYSTEMS USING LINEAR LYAPUNOV FUNCTIONALS AND A POSITIVE SYSTEMS APPROACH}

\author{
Frédéric Mazenc \\ EPI DISCO INRIA-Saclay, L2S \\ CNRS-Supelec, 3 rue Joliot Curie \\ 91192 Gif-sur-Yvette, France \\ Email: Frederic.Mazenc@Iss.supelec.fr
}

\author{
Michael Malisoff * \\ Department of Mathematics \\ Louisiana State University \\ Baton Rouge, Louisiana 70803-4918 \\ Email: malisoff@Isu.edu
}

\begin{abstract}
We present new methods for proving stability of time-varying linear systems with delays. Our main tools include positive systems and linear Lyapunov functionals. Our work applies to key classes of systems that arise in numerous engineering applications, including neutral systems, and systems that are not necessarily periodic in time and not necessarily positive. We prove stability by comparing the trajectories of the original systems with trajectories of higher dimensional positive systems. One of our key results requires an upper bound on the delay, but the delay can be unknown. Our work also provides robustness of the stability with respect to uncertainties in the coefficient matrices of the system. We illustrate our work in three examples, which show how our methods can sometimes be used with backstepping and linearization to cover even more general classes of systems.
\end{abstract}

\section{INTRODUCTION}

The study of stability and control methods for time-varying systems with delay is motivated by the fact that delays often arise from measurement and transport phenomena in networked control, population dynamics, and many other settings [1-3], which lead to time-varying systems when one must track a desired reference trajectory. Neutral systems are an important class of delay systems in which time delays occur in the time derivatives of the state components [4,5]. Many important systems can be modeled by neutral systems, such as distributed networks [6], heat exchangers [7], and population models [8, Chap. 2]. How-

${ }^{*}$ Address all correspondence to this author. ever, proving stability for such systems can be difficult, because the usual classical techniques do not apply. For example, while frequency domain approaches are useful for time invariant neutral systems [4], neither frequency domain approaches nor linear matrix inequality techniques apply to time-varying systems with delays. Although there are many significant stability results for time-varying systems with delays (such as [9]), applying them often requires Lyapunov functions that may not always be easy to find, and Lyapunov function constructions based on linear matrix inequalities for time invariant systems have no clear analog for time-varying systems. Therefore, despite their importance in engineering applications, there are significant remaining challenges for establishing stability properties for time-varying systems with delays.

The works $[10,11]$ use a different approach to studying stability of time delay systems that was based on nonnegative systems and linear Lyapunov functions. The linearity property contrasts with the usual definitions of Lyapunov functions, which require the Lyapunov functions to be everywhere nonnegative, and may at first seem surprising, given that Lyapunov-type functionals usually have the null function as the linear approximation at the origin [9], [12], [13], [14], [15]. By nonnegativity of a system, we mean that for all admissible componentwise nonnegative valued initial functions for the system, all components of the solutions stay nonnegative for all nonnegative times. See below for all of our definitions. One contribution of this note is to extend the linear Lyapunov function approach from $[10,11]$ to neutral systems that are not necessarily nonnegative, by reducing the analysis to the study of systems on the nonnegative orthant. 
A second contribution of this note is to use analogs of the methods [16] for neutral systems to provide a stability analysis method for linear time-varying systems with pointwise delays. Our work for linear time-varying systems does not require that the vector fields be periodic in time, and has four important ingredients. The first involves representing the system as a system with a distributed delay, coupled with an integral equation. Next, we show that all trajectories of the coupled system are components of trajectories of a higher dimensional positive system. The last step finds a linear Lyapunov-Krasovskii functional for the higher dimensional system. Also, we take the potentially stabilizing effects of the delayed term into account, which were not taken into account in [16]. Our linear Lyapunov functional construction bears similarities to the time invariant ones in [10] for time invariant systems; see also [11] and [17].

While much of the existing delays literature (such as [18] and [19]) is concerned with delays in the input, we do not require such a structure here. Prediction is a powerful approach to designing controllers that compensate arbitrarily long input delays $[1,2,20]$, but it may not always lend itself to cases where the delays occur in the vector fields of the system. Instead of prediction or other standard methods, we decompose certain functions into cooperative and non-cooperative parts, which is analogous to the methods in [17], [21], and [22] for time invariant systems or interval observers. Our decomposition is related to the internal positive representation in [23] and [24]. Two practical advantages of our new technique are that (a) its assumptions seem readily checkable and (b) it leads to exponential stability results for systems for which no other techniques seem to apply. One theorem we present here uses a bound on the delay, but the actual delay value may be unknown.

This note is a summary of some of our work in $[16,25,26]$, which have complete proofs. The work [26] contains theory that generalizes [25] to cases where the coefficient matrices in the linear system can be uncertain, or where they also depend on past values of the state. In the next section, we provide the definitions needed to make the statements of our results precise. Then we state the key lemmas and the main result from [16], and then we summarize some of our findings from $[25,26]$ and present examples that illustrate our results. We close by summarizing our work and suggesting future research topics.

\section{DEFINITIONS AND NOTATION}

Throughout what follows, the dimensions of our vectors and matrices are arbitrary positive integers, unless otherwise noted. We set $\mathbb{R}=(-\infty,+\infty)$ and $\mathbb{N}=\{1,2, \ldots$,$\} , and \mathbb{R}^{p \times q}$ is the set of all $p \times q$ matrices with real entries. For each matrix $M \in \mathbb{R}^{p \times q}$, let $m_{i j}$ be its entry in row $i$ and column $j$ for all $i$ and $j$. We use 0 to denote the zero matrix, and $|\cdot|$ denotes the usual Euclidean norm of vectors and the induced matrix norm, in any dimensions. All inequalities are meant componentwise, i.e., for any vectors $v_{a}=\left(v_{a 1}, \ldots, v_{a r}\right)^{\top} \in \mathbb{R}^{r}$ and $v_{b}=\left(v_{b 1}, \ldots, v_{b r}\right)^{\top} \in \mathbb{R}^{r}$, the symbol $v_{a} \leq v_{b}$ means that $v_{a i} \leq v_{b i}$ for all $i \in\{1, \ldots, r\}$. A square matrix is cooperative or Metzler provided all of its off-diagonal entries are nonnegative. We call a matrix $M \in \mathbb{R}^{r \times s}$ nonnegative (resp., positive) provided each of its entries $m_{i j}$ satisfies $m_{i j} \geq 0$ (resp., $>0$ ). We define negative and nonpositive matrices analogously, by reversing the inequalities. To simplify notation, we always take the initial times for our trajectories to be $t_{0}=0$.

We use $M^{+}$to denote the matrix whose position $(i, j)$ entry is $\max \left\{0, m_{i j}\right\}$ for all $i$ and $j$, and we set $M^{-}=M^{+}-M$. We use $\bar{M}$ to denote the matrix whose diagonal entries are $m_{i i}$ and whose off-diagonal entries are $\max \left\{0, m_{i j}\right\}$, and we set $\underline{M}=\bar{M}-M$. We let $M^{s}=M^{+}+M^{-}$, so $M^{s}=\left[\left|m_{i j}\right|\right]$. We also set $M^{*}=\bar{M}+\underline{M}$. Then, $M^{*}$ is always Metzler. A matrix $A$ is called Schur stable provided its spectral radius is strictly smaller than 1 .

We use $C^{1}$ to denote the set of all continuously differentiable functions, whose domains and ranges will be clear from the context. For any constant $\tau>0$, let $C\left([-\tau, 0], \mathbb{R}^{n}\right)$ denote the set of all continuous $\mathbb{R}^{n}$-valued functions defined on $[-\tau, 0]$. This set is abbreviated by $C_{\text {in }}(\tau)$ or simply $C_{\text {in }}$, and is called the set of all initial functions. A system is called positive (resp.,nonnegative) for a class of initial functions $C_{0}$ provided for each positive valued initial function in $C_{0}$, the unique solution of the system stays positive (resp., nonnegative) for all $t \geq 0$. Given any continuous function $\varphi:[-\tau,+\infty) \rightarrow \mathbb{R}^{n}$ and any $t \geq 0$, we define $\varphi_{t} \in C_{\text {in }}$ by $\varphi_{t}(m)=\varphi(t+m)$ for all $m \in[-\tau, 0]$.

For any real numbers $a$ and $b>a$, a function $\psi:[a, b) \rightarrow \mathbb{R}^{n}$ is called piecewise continuous provided for any constant $c \in$ $(a, b), \psi$ is continuous over $[a, c)$, except at a finite number of points. The solution of a time delay system described by a functional differential equation

$$
\dot{X}(t)=\mathcal{F}\left(t, \dot{X}(t-\tau), X_{t}\right)
$$

with a $C^{1}$ initial function $\phi x \in C_{\text {in }}$ is denoted by $X(t)$, instead of by $\mathcal{X}\left(t, t_{0}, \phi_{\chi}\right)$ as more formally done for instance in [9]. More generally, the notation will be simplified whenever no confusion would arise. In particular, the time derivative of a Lyapunov functional $V\left(t, X_{t}\right)$ along all trajectories of the system in Eqn. (1) will be denoted by $\dot{V}(t)$.

\section{PRELIMINARY RESULTS FROM [16]}

This section summarizes the preliminary results from [16] that are needed to prove our main result for neutral systems in the next section. Our preliminary results are also of independent interest. Consider the interconnection

$$
\left\{\begin{array}{c}
\dot{\xi}(t)=H(t) \xi(t)+P_{1}(t) \xi\left(t-\tau_{2}\right)+P_{2}(t) \psi\left(t-\tau_{1}\right) \\
\psi(t)=P_{3}(t) \psi\left(t-\tau_{1}\right)+P_{4}(t) \xi(t)+P_{5}(t) \xi\left(t-\tau_{2}\right)
\end{array}\right.
$$

of a differential equation and a static system, where $\xi$ is valued in $\mathbb{R}^{m}, \psi$ is valued in $\mathbb{R}^{l}, \tau_{1}>0$ and $\tau_{2} \geq 0$ are constant delays, and the matrices $P_{i}$ for $i=1$ to 5 and $H$ are continuous. Set $\tau_{M}=\max \left\{\tau_{1}, \tau_{2}\right\}$. Our first assumption on Eqns. (2) is: 
Assumption 1. For all $t \geq 0$, the matrix $H(t)$ is Metzler and the matrices $P_{i}(t)$ for $i=1$ to 5 are nonnegative. Also, there is a constant $c_{a}>0$ such that for all $t \geq 0$, the inequality

$$
|H(t)|+\sum_{j=1}^{5}\left|P_{j}(t)\right| \leq c_{a}
$$

is satisfied.

In [16], the following useful observation is shown:

Lemma 1. Let Eqns. (2) satisfy Assumption 1. Let $\left(\phi_{\xi}, \phi_{\psi}\right) \in$ $C_{\text {in }}\left(\tau_{M}\right)$ be positive valued. Let $(\xi, \psi)$ be any solution of Eqns. (2) such that $\xi(t)=\phi_{\xi}(t)$ and $\psi(t)=\phi_{\psi}(t)$ hold for all $t \in$ $\left[-\tau_{M}, 0\right)$. Then, $(\xi, \psi)$ is piecewise continuous over $\left[-\tau_{M},+\infty\right)$, and $\xi(t)>0$ and $\psi(t)>0$ hold for all $t \geq-\tau_{M}$. Moreover, there are no other solutions to Eqns. (2) with the same initial conditions. Also, $\xi$ is continuous.

Lemma 1 follows by induction, by showing that for all integers $k \geq 0$, the solution is nonnegative and uniquely defined on $\left[-\tau_{M}, k \tau_{1}\right)$, and piecewise continuous over $\left[(j-1) \tau_{1}, j \tau_{1}\right)$ for all $j \in\{0, \ldots, k\}$. The following lemma from [16] is also used:

Lemma 2. Let $\tau>0$ be any positive constant. Let $g$ : $[-\tau,+\infty) \rightarrow[0,+\infty)$ be any function that is locally bounded, continuous on $[-\tau, 0)$, and continuous on each interval $[i \tau,(i+$ 1) $\tau)$ for all $i \geq 0$. Let $\xi:[0,+\infty) \rightarrow(0,+\infty)$ be any continuous nonincreasing function such that $\lim _{t \rightarrow+\infty} \zeta(t)=0$. Let $\varepsilon \in(0,1)$ be any constant such that the inequality $g(t) \leq \varepsilon g(t-\tau)+\zeta(t)$ is satisfied for all $t \geq \tau$. Then $\lim _{t \rightarrow+\infty} g(t)=0$.

Next, we add:

Assumption 2. There exist a $C^{1}$ function $\varpi:[0,+\infty) \rightarrow \mathbb{R}^{m}$; constant column vectors $\gamma \in \mathbb{R}^{l}, \varpi_{a} \in \mathbb{R}^{m}$, and $\varpi_{b} \in \mathbb{R}^{m}$ such that $\gamma>0, \varpi_{a}>0$, and $\varpi_{b}>0$ hold; a vector $\rho_{0}>0$; and constant real numbers $\delta \in(0,1)$ and $c_{b}>0$, such that with the choices

$$
\begin{aligned}
\rho_{1}(t)= & -\left[\dot{\varpi}(t)^{\top}+\varpi(t)^{\top} H(t)+\gamma^{\top} P_{4}(t)\right. \\
& \left.+\varpi\left(t+\tau_{2}\right)^{\top} P_{1}\left(t+\tau_{2}\right)+\gamma^{\top} P_{5}\left(t+\tau_{2}\right)\right], \text { and } \\
\rho_{2}(t)= & -\left[\varpi(t)^{\top} P_{2}(t)+\gamma^{\top} P_{3}(t)-\gamma^{\top}\right],
\end{aligned}
$$

the following are satisfied:

$$
\begin{gathered}
|\dot{\varpi}(t)| \leq c_{b}, \\
\varpi_{a}<\varpi(t)<\varpi_{b}, \\
\gamma^{\top} P_{3}(t) \leq \delta \gamma^{\top}, \\
\rho_{1}(t) \geq \rho_{0}, \text { and } \rho_{2}(t) \geq 0
\end{gathered}
$$

hold for all $t \in[0,+\infty)$.

The motivation for Assumption 2 is as follows. Recall the following two results from [10, Chapt. 2]: (A) A constant matrix $H$ is Metzler and Hurwitz if and only if there exist constant vectors $\varpi_{1}>0$ and $\varpi_{2}>0$ such that $\varpi_{1}^{\top} H+\varpi_{2}^{\top}=0$. (B) A nonnegative constant matrix $M$ is Schur stable if and only if there exist a constant vector $\varpi_{3}>0$ and a constant $c_{d} \in(0,1)$ such that $\boldsymbol{\varpi}_{3}^{\top} M \leq c_{d} \varpi_{3}^{\top}$. Assumption 2 holds in this special case with $P_{3}=M$ and the other $P_{i}$ 's taken to be zero. Assumption 2 is a generalization that applies to time-varying cases. If $H$ is periodic, then we can find a time-varying change of coordinates to transform a nonconstant matrix $H$ into a constant matrix $H$, which may facilitate the determination of a function $\varpi$ to satisfy Assumption 2. We also need the following result from [16]:

Lemma 3. Assume that Eqns. (2) satisfies Assumptions 1 and 2. Then for any pair $\left(\phi_{\xi}, \phi_{\psi}\right) \in C_{\mathrm{in}}\left(\tau_{M}\right)$ and any solution $(\xi(t), \psi(t))$ of Eqns. (2) such that $\xi(t)=\phi_{\xi}(t)$ and $\psi(t)=\phi_{\psi}(t)$ hold for all $t \in\left[-\tau_{M}, 0\right)$, we have $\lim _{t \rightarrow \infty}(\xi(t), \psi(t))=0$.

The strategy for proving Lemma 3 is to use the linear function that is defined along all trajectories of Eqns. (2) by

$$
\begin{aligned}
& U_{2}\left(t, \xi_{t}, \psi_{t}\right)=U_{1}\left(t, \xi_{t}, \psi_{t}\right) \\
& +\int_{t-\tau_{2}}^{t}\left[\varpi\left(l+\tau_{2}\right)^{\top} P_{1}\left(l+\tau_{2}\right)+\gamma^{\top} P_{5}\left(l+\tau_{2}\right)\right] \xi(l) \mathrm{d} l,
\end{aligned}
$$

where

$$
U_{1}\left(t, \xi_{t}, \psi_{t}\right)=\varpi(t)^{\top} \xi(t)+\int_{t-\tau_{1}}^{t} \gamma^{\top} \psi(m) \mathrm{d} m,
$$

in conjunction with Barbalat's Lemma; see [16] for details.

\section{DISCUSSION OF MAIN RESULT FROM [16]}

The main result in [16] gives conditions that ensure stability properties of time-varying neutral systems of the form

$$
\dot{x}(t)-L(t) \dot{x}\left(t-\tau_{1}\right)=M(t) x(t)+N(t) x\left(t-\tau_{2}\right),
$$

where $x$ is valued in $\mathbb{R}^{m}, \tau_{1}>0$ and $\tau_{2} \geq 0$ are constant delays, and the functions $L, M$ and $N$ are continuous. We say that a function $x$ with a $C^{1}$ initial condition is a solution of Eqn. (11) provided the following hold for all $j \in \mathbb{N}$ : (a) the function $x$ is of class $C^{1}$ over $\left[(j-1) \tau_{1}, j \tau_{1}\right)$ and (b) $x$ satisfies Eqn. (11) for all $t \in\left((j-1) \tau_{1}, j \tau_{1}\right)$. We introduce the following assumption:

Assumption 3. There exist a constant $c_{g}>0$; a positive valued $C^{1}$ function $p:[0,+\infty) \rightarrow \mathbb{R}^{m}$; constant vectors $q>0$, $p_{a}>0, p_{b}>0, r_{1,0}>0$ and $r_{2,0} \geq 0$ of appropriate dimensions; and a constant $\varsigma \in(0,1)$, such that with the choices

$$
\begin{aligned}
r_{1}(t)= & -\left[\dot{p}(t)+p(t)^{\top} M^{*}(t)+q^{\top} M^{s}(t)\right. \\
& \left.+\left(p\left(t+\tau_{2}\right)+q\right)^{\top} N^{s}\left(t+\tau_{2}\right)\right] \text { and } \\
r_{2}(t)= & -\left[p(t)^{\top} L^{s}(t)+q^{\top}\left(L^{s}(t)-I_{m}\right)\right],
\end{aligned}
$$

the following conditions hold for all $t \geq 0$ :

$$
\begin{gathered}
|L(t)|+|M(t)|+|N(t)| \leq c_{g}, \\
r_{1}(t) \geq r_{1,0}, r_{2}(t) \geq r_{2,0}, \\
q^{\top} L^{s}(t) \leq \varsigma q^{\top}, \text { and } \\
p_{a} \leq p(t) \leq p_{b} .
\end{gathered}
$$

Also, $\dot{p}$ is bounded. 
The main result from [16] is then:

Theorem 1. Assume that the system in Eqn. (11) satisfies Assumption 3. Then all solutions of Eqn. (11) for all initial functions in $C_{\text {in }}$ of class $C^{1}$ converge to the origin as $t \rightarrow+\infty$.

For the proof of Theorem 1, see [16]. Notice that Assumption 3 does not put any sign restrictions on the components of $L, M$, and $N$. Also, the solutions of our neutral system are not necessarily $C^{1}$ over $\left[-\tau_{M},+\infty\right)$, but they are $C^{1}$ over the intervals $\left((j-1) \tau_{1}, j \tau_{1}\right)$ for all $j \in \mathbb{N}$. On the other hand, even if $M$ is a constant Hurwitz matrix $M_{c}$, the corresponding matrix $M_{c}^{*}$ may not be Hurwitz; see [27] and [28]. To overcome this potential obstacle to applying Theorem 1, it may be useful to apply the (possibly time-varying) changes of coordinates [28], to try to transform a time invariant linear system into a cooperative one.

\section{DISCUSSION OF MAIN RESULT FROM [25]}

Our main result from [25] provides a stability analysis for linear time-varying systems of the form

$$
\dot{x}(t)=A_{1}(t) x(t)+A_{2}(t) x(t-\tau),
$$

where $x$ is valued in $\mathbb{R}^{n}, \tau \geq 0$ is the constant delay, the initial functions are in $C_{\text {in }}(\tau)$, and $A_{1}:[0,+\infty) \rightarrow \mathbb{R}^{n \times n}$ and $A_{2}$ : $[0,+\infty) \rightarrow \mathbb{R}^{n \times n}$ are continuous functions, but see our examples section below where we leverage our results for Eqn. (17) to cover more general systems, using linearizations and backstepping. Also, see [26] and the remarks below for generalizations to systems where $A_{1}$ and $A_{2}$ have multiplicative uncertainties and where they can also depend on $x_{t}$. Eqn. (17) covers time-varying linear systems with linear feedbacks with input delays, and linearizations of nonlinear systems around reference trajectories, in closed loop with delayed linear controls.

To simplify the statements of our results, we define

$$
\begin{gathered}
B_{1}(t)=A_{1}(t)+A_{2}(t+\tau), B_{2}(t)=-A_{2}(t+\tau), \\
\text { and } B_{3}(t, m)=B_{1}(t) B_{2}(m) .
\end{gathered}
$$

We use the following assumptions from [25]:

Assumption 4. The matrix $A_{1}$ is bounded, and there are constants $\mathfrak{c}_{j}>0$ and a $C^{1}$ function $p:[0, \infty) \rightarrow \mathbb{R}^{n}$ such that

$$
\begin{gathered}
\dot{p}(t)^{\top}+p(t)^{\top}\left(\bar{B}_{1}(t)+\underline{B}_{1}(t)\right) \leq-\mathfrak{c}_{1} p(t)^{\top}, \\
\mathfrak{c}_{2}(1 \ldots 1)^{\top} \leq p(t) \leq \mathfrak{c}_{3}(1 \ldots 1)^{\top}, \\
p^{\top}(t) B_{2}^{s}(t) \leq \mathfrak{c}_{4} p^{\top}(t), \text { and } \\
p^{\top}(t) B_{3}^{s}(t, m) \leq \mathfrak{c}_{6} p^{\top}(t)
\end{gathered}
$$

for all $t \geq 0$ and $m \geq 0$.

Setting $\mathfrak{c}_{5}=\mathfrak{c}_{3} \mathfrak{c}_{4} / \mathfrak{c}_{2}$, we also assume:

Assumption 5. The delay $\tau$ satisfies

$$
\left(\frac{\mathfrak{c}_{6}}{\mathfrak{c}_{1}}+\mathfrak{c}_{5}\right) \tau<1
$$

where the $\mathfrak{c}_{i}$ 's are from Assumption 4.
We can then prove:

Theorem 2. If the system in Eqn. (17) satisfies Assumptions 4-5, then it is uniformly globally exponentially stable to 0.

Before summarizing the proof of Theorem 2, we make several observations. When $B_{1}^{*}$ is constant and Hurwitz, we can find a vector $p_{0}>0$ such that inequality (20) holds with $p(t)=p_{0}$ for all $t \geq 0$ [10]. Using a time-varying change of coordinates, we can sometimes transform $\dot{X}=B_{1}(t) X$ into an autonomous system [29], [30], which may help produce a function $p$ that satisfies Assumption 4. Assumption 5 restricts the size of the delay $\tau$, but it does not require that $\tau$ is known. As we will see in an example below, Assumptions 4-5 do not imply that $\dot{X}=A_{1}(t) X$ is asymptotically stable. One important difference between [16] and Theorem 2 is that the potential stabilizing effect of the term $A_{2}(t) x(t-\tau)$ is not taken into account in [16], while Theorem 2 takes that effect into account; see the examples section below.

We next summarize the main steps in the proof of Theorem 2 in [25]. Using the time-varying operator

$$
\xi(t)=x(t)+\int_{t-\tau}^{t} A_{2}(m+\tau) x(m) \mathrm{d} m
$$

and the notation above, we can rewrite (17) as [25]

$$
\left\{\begin{aligned}
\dot{\xi}(t)= & \bar{B}_{1}(t) \xi(t)-\underline{B}_{1}(t) \xi(t)+\int_{t-\tau}^{t} B_{3}^{+}(t, m) x(m) \mathrm{d} m \\
& -\int_{t-\tau}^{t} B_{3}^{-}(t, m) x(m) \mathrm{d} m \\
x(t)= & \xi(t)+\int_{t-\tau}^{t} B_{2}^{+}(m) x(m) \mathrm{d} m-\int_{t-\tau}^{t} B_{2}^{-}(m) x(m) \mathrm{d} m .
\end{aligned}\right.
$$

Then we embed all trajectories ( $\xi, x)$ of Eqns. (24) as solutions of the relevant subsystem of the higher dimensional system

$$
\left\{\begin{aligned}
\dot{\xi}(t)= & \bar{B}_{1}(t) \xi(t)+\underline{B}_{1}(t) \Psi(t) \\
& +\int_{t-\tau}^{t} B_{3}^{+}(t, m) x(m) \mathrm{d} m \\
& +\int_{t-\tau}^{t} B_{3}^{-}(t, m) Z(m) \mathrm{d} m \\
x(t)= & \xi(t)+\int_{t-\tau}^{t} B_{2}^{+}(m) x(m) \mathrm{d} m \\
& +\int_{t-\tau}^{t} B_{2}^{-}(m) Z(m) \mathrm{d} m \\
\dot{\Psi}(t)= & \bar{B}_{1}(t) \Psi(t)+\underline{B}_{1}(t) \xi(t) \\
& +\int_{t-\tau}^{t} B_{3}^{+}(t, m) Z(m) \mathrm{d} m \\
& +\int_{t-\tau}^{t} B_{3}^{-}(t, m) x(m) \mathrm{d} m \\
Z(t)= & \Psi(t)+\int_{t-\tau}^{t} B_{2}^{+}(m) Z(m) \mathrm{d} m \\
& +\int_{t-\tau}^{t} B_{2}^{-}(m) x(m) \mathrm{d} m
\end{aligned}\right.
$$

by choosing $Z(t)=-x(t)$ and $\Psi(t)=-\xi(t)$, where we also require the matching condition

$$
\begin{aligned}
& x(0)=\xi(0)+\int_{-\tau}^{0} B_{2}^{+}(m) x(m) \mathrm{d} m+\int_{-\tau}^{0} B_{2}^{-}(m) Z(m) \mathrm{d} m \\
& Z(0)=\Psi(0)+\int_{-\tau}^{0} B_{2}^{+}(m) Z(m) \mathrm{d} m+\int_{-\tau}^{0} B_{2}^{-}(m) x(m) \mathrm{d} m .
\end{aligned}
$$

This means that $(\xi, x,-\xi,-x)$ is a solution of Eqns. (25) exactly when $(\xi, x)$ is a solution of Eqns. (24). Then we can show that all trajectories of the original system satisfy the required exponential stability condition, provided all everywhere componentwise positive solutions of Eqns. (25)-(26) satisfy an exponential stability condition. Hence, the rest of the proof entails proving an 
exponential stability estimate for solutions of Eqns. (25)-(26). This is done by setting $c(t)=x(t)+Z(t)$ and $\gamma(t)=\xi(t)+\Psi(t)$, and using the linear Lyapunov-Krasovskii functional

$$
\begin{aligned}
& V_{3}\left(t, \gamma(t), c_{t}\right)=V_{1}(t, \gamma(t))+h V_{2}\left(t, c_{t}\right), \text { where } \\
& V_{1}(t, \gamma)=p(t)^{\top} \gamma, \quad p(t) \text { is from Assumption } 4, \\
& V_{2}\left(t, c_{t}\right)=\int_{t-\tau}^{t}(g-t+\ell) p^{\top}(\ell) c(\ell) \mathrm{d} \ell,
\end{aligned}
$$

and $g>0$ and $h>0$ are suitable constants. In fact, by choosing appropriate $g$ and $h$, we can find a positive constant $\mathfrak{c}_{7}$ such that

$$
\begin{aligned}
& \mathfrak{c}_{2} \sum_{i=1}^{n} \gamma_{i}\left(t_{1}\right)+h(g-\tau) \mathfrak{c}_{2} \sum_{i=1}^{n} \int_{t_{1}-\tau}^{t_{1}} c_{i}(\ell) \mathrm{d} \ell \leq \\
& e^{-\mathfrak{c}_{7}\left(t_{1}-t_{2}\right)}\left[V_{1}\left(t_{2}, \gamma\left(t_{2}\right)\right)+h V_{2}\left(t_{2}, c_{t_{2}}\right)\right]
\end{aligned}
$$

holds along all trajectories of the $(\gamma, c)$ system, which leads to the desired exponential stability for the higher dimensional system and so also for the original system. See [25] for more details.

Remark 1. We can generalize Theorem 2 to prove global asymptotic stability of systems of the form

$$
\dot{x}(t)=\left(\delta A_{1}\right)\left(t, x_{t}\right) x(t)+\left(\delta A_{2}\right)\left(t, x_{t}\right) x(t-\tau)
$$

where the $(i, j)$ entry of $\left(\delta A_{p}\right)\left(t, x_{t}\right)$ is $\delta_{p i j}\left(t, x_{t}\right) a_{p i j}\left(t, x_{t}\right)$ for all $i$ and $j$ in $\{1,2, \ldots, n\}$, the functions $A_{p}=\left[a_{p i j}\right]:[0, \infty) \times C_{\mathrm{in}} \rightarrow$ $\mathbb{R}^{n \times n}$ are known for $p=1,2$, and the functions $\delta_{\text {pij }}:[0, \infty) \rightarrow$ $\left[\delta_{*}, \delta^{*}\right]$ are continuous uncertainties whose constant bounds $\delta_{*}>$ 0 and $\delta^{*}>0$ are known. The works [16, 25] did not cover systems with uncertainties of the form (29). The main change in our assumptions from [25] needed to cover (29) is to replace (22) by

$$
\delta^{*}\left(\delta^{*} \frac{\mathfrak{c}_{6}}{\mathfrak{c}_{1}}+\mathfrak{c}_{5}\right) \tau<1,
$$

where the $\mathfrak{c}_{i}$ 's are defined analogously to the constants in Assumptions 4-5. See [26] for the proof of this generalized result.

\section{EXAMPLES}

We next illustrate our theorems by proving results that we believe do not follow from any standard results. In our first two examples, our theorems apply directly. Then, we illustrate how we can combine our theorems with backstepping and linearization to cover even more general systems. The following examples are from $[16,25]$, but see the remarks at the end of this section for a generalization that is not found in $[16,25]$.

\section{First Example: Neutral System}

We illustrate Theorem 1 with the one dimensional system

$$
\dot{x}(t)=\eta a(t) \dot{x}(t-\tau)-2 x(t)+b(t) x(t-\tau),
$$

where $x$ is valued in $\mathbb{R}$, the continuous functions $a$ and $b$ are valued in $[-1,1]$, and $\tau>0$ and $\eta \in(0,1)$ are constants. Due to the terms $\eta a(t) \dot{x}(t-\tau)$ and $b(t) x(t-\tau)$, this system is not nonnegative (e.g., because the zero initial function produces negative values for $x(t)$ for $t>0)$. We study the stability of Eqn. (31). Using the notation of Theorem 1 , we take $L(t)=\eta a(t), M(t)=-2$,
$N(t)=b(t)$, and $q=1$. Then (12) becomes

$$
\begin{aligned}
r_{1}(t)= & -[\dot{p}(t)-2 p(t)+2 \\
& +(p(t+\tau)+1)|b(t+\tau)|] \text { and } \\
r_{2}(t)= & -p(t) \eta|a(t)|+1-\eta|a(t)| .
\end{aligned}
$$

We choose the constant function $p(t)=\frac{1}{\eta}-1$. Then (32) become

$$
r_{1}(t)=2\left(\frac{1}{\eta}-2\right)+\frac{1}{\eta}|b(t+\tau)| \text { and } r_{2}(t)=1-|a(t)| .
$$

Since $r_{1}(t) \geq 2((1 / \eta)-2)-(1 / \eta)=(1 / \eta)-4$ and $L^{s}(t)=$ $\eta|a(t)| \leq \eta$ hold for all $t \geq 0$, we can conclude from Theorem 1 that all solutions of the system in Eqn. (31) converge to zero as $t \rightarrow+\infty$, when $\eta \in(0,1 / 4)$.

\section{Second Example: Scalar Time-Varying System}

Take the one dimensional system

$$
\dot{x}(t)=\ell_{1} \cos ^{2}(t) x(t)+\ell_{2} \sin (t) x(t-\tau),
$$

where $\tau \geq 0, \ell_{1} \in \mathbb{R}$, and $\ell_{2} \in \mathbb{R}$ are constants. We use Theorem 2 to find conditions on $\tau, \ell_{1}$ and $\ell_{2}$ that ensure that Eqn. (34) is uniformly globally exponentially stable to 0 . Using the notation from Theorem 2 , we have $B_{1}(t)=l_{1} \cos ^{2}(t)+l_{2} \sin (t+\tau)$, $B_{2}(t)=-l_{2} \sin (t+\tau), B_{1}^{*}(t)=B_{1}(t)$, and $B_{3}(t, m)=-l_{2} \sin (m+$ $\tau)\left(l_{1} \cos ^{2}(t)+l_{2} \sin (t+\tau)\right)$.

We need a $C^{1}$ function $p:[0,+\infty) \rightarrow \mathbb{R}$ and a constant $\mathfrak{c}_{1}>0$ such that $\dot{p}(t)+p(t) B_{1}(t) \leq-\mathfrak{c}_{1} p(t)$ for all $t \geq 0$. Assume that $\ell_{1}<0$ and choose $\mathfrak{c}_{1}=-\ell_{1} / 2$. Then, the double angle formula allows us to rewrite our requirement as $\dot{p}(t) \leq\left[-\ell_{1} \cos (2 t) / 2-\ell_{2} \sin (t+\tau)\right] p(t)$, so we can take $p(t)=$ $\exp \left(-\ell_{1} \sin (2 t) / 4+\ell_{2} \cos (t+\tau)\right)$. Hence, Assumptions 45 hold if we take $\mathfrak{c}_{2}=\exp \left(-\left|l_{1}\right| / 4-\left|l_{2}\right|\right), \mathfrak{c}_{3}=1 / \mathfrak{c}_{2}, \mathfrak{c}_{5}=$ $\left|l_{2}\right| \exp \left(\left|l_{1}\right| / 2+2\left|l_{2}\right|\right), \mathfrak{c}_{6}=\left(\left|l_{1}\right|+\left|l_{2}\right|\right)\left|l_{2}\right|$, and all $\tau>0$ such that

$$
\left|l_{2}\right|\left(\frac{2}{\left|l_{1}\right|}\left(\left|l_{1}\right|+\left|l_{2}\right|\right)+e^{0.5\left|l_{1}\right|+2\left|l_{2}\right|}\right) \tau<1 .
$$

By Theorem 2, we conclude that Eqn. (34) is uniformly globally exponentially stable to 0 , when $\ell_{1}<0$ and (35) hold.

\section{Third Example: Stabilizing Term with Delay}

For another example that illustrates Theorem 2, consider

$$
\left\{\begin{array}{l}
\dot{\xi}_{1}(t)=v_{1}(t-\tau) \\
\dot{\xi}_{2}(t)=v_{2}(t-\tau) \\
\dot{\xi}_{3}(t)=\xi_{2}(t) v_{1}(t),
\end{array}\right.
$$

where we assume that the constant delay $\tau$ satisfies $\tau \in(0,1 /(3+$ $2 \sqrt{e})$ ). Our work [29, Section 6.2] solved a tracking problem for Eqns. (36) for the reference trajectory $(-\cos (t), 0,0)^{\top}$ when $\tau=0$, by building a strict Lyapunov function for the tracking dynamics. However, [29] was limited to undelayed systems, and there is no clear analog of [29, Section 6.2] that can be applied to delayed systems. Therefore, we use Theorem 2 to solve the problem of locally exponentially tracking the trajectory $(\sin (t), 0,0)^{\top}$ when $\tau \in(0,1 /(3+2 \sqrt{e}))$. By choosing $\gamma_{1}(t)=\xi_{1}(t)-\sin (t)$ 
and $v_{1}(t)=\cos (t+\tau)-\gamma_{1}(t)$, we obtain

$$
\left\{\begin{array}{l}
\dot{\gamma}_{1}(t)=-\gamma_{1}(t-\tau) \\
\dot{\xi}_{2}(t)=v_{2}(t-\tau) \\
\dot{\xi}_{3}(t)=\xi_{2}(t)\left[\cos (t+\tau)-\gamma_{1}(t)\right] .
\end{array}\right.
$$

Since $\tau \in(0,1 / 3)$, the system $\dot{\gamma}_{1}(t)=-\gamma_{1}(t-\tau)$ is globally exponentially stable to 0 (GES) [25].

The linear approximation of Eqns. (37) at 0 is

$$
\left\{\begin{array}{l}
\dot{\xi}_{2}(t)=v_{2}(t-\tau) \\
\dot{\xi}_{3}(t)=\xi_{2}(t) \cos (t+\tau) \\
\dot{\gamma}_{1}(t)=-\gamma_{1}(t-\tau)
\end{array}\right.
$$

Hence, the origin of Eqns. (38) is GES provided the origin of

$$
\left\{\begin{array}{l}
\dot{\xi}_{2}(t)=v_{2}(t-\tau) \\
\dot{\xi}_{3}(t)=\cos (t+\tau) \xi_{2}(t)
\end{array}\right.
$$

is GES. To show the GES property for Eqns. (39), we set $\gamma_{2}(t)=$ $\xi_{2}(t)+\cos (t+\tau) \xi_{3}(t-\tau)$ to transform Eqns. (39) into

$$
\left\{\begin{aligned}
\dot{\xi}_{3}(t)= & -\cos ^{2}(t+\tau) \xi_{3}(t-\tau)+\cos (t+\tau) \gamma_{2}(t) \\
\dot{\gamma}_{2}(t)= & v_{2}(t-\tau)-\sin (t+\tau) \xi_{3}(t-\tau) \\
& +\cos (t+\tau)\left[-\cos ^{2}(t) \xi_{3}(t-2 \tau)\right. \\
& \left.+\cos (t) \gamma_{2}(t-\tau)\right]
\end{aligned}\right.
$$

Taking

$$
\begin{aligned}
& v_{2}(t-\tau)=\sin (t+\tau) \xi_{3}(t-\tau)-\gamma_{2}(t-\tau) \\
& -\cos (t+\tau)\left[-\cos ^{2}(t) \xi_{3}(t-2 \tau)+\cos (t) \gamma_{2}(t-\tau)\right]
\end{aligned}
$$

gives the triangular system

$$
\left\{\begin{array}{l}
\dot{x}_{1}(t)=-\cos ^{2}(t+\tau) x_{1}(t-\tau)+\cos (t+\tau) x_{2}(t) \\
\dot{x}_{2}(t)=-x_{2}(t-\tau)
\end{array}\right.
$$

where $x_{1}=\xi_{3}$ and $x_{2}=\gamma_{2}$. Using the preceding backstepping approach, we now use Theorem 2 to study the stability properties of the system (42). With the notation (18), we get

$$
B_{1}^{*}(t)=\left[\begin{array}{cc}
-\cos ^{2}(t+2 \tau) & |\cos (t+\tau)| \\
0 & -1
\end{array}\right] .
$$

Choose the function $p: \mathbb{R} \rightarrow \mathbb{R}^{2}$ defined by $p(t)=$ $\left(e^{\sin (2 t+4 \tau) / 4}, 2 e^{1 / 4}\right)^{\top}$. Then

$$
\dot{p}(t)=\left[\cos (2 t+4 \tau) e^{\frac{1}{4} \sin (2 t+4 \tau)} / 2 \quad 0\right]^{\top} .
$$

Then we can show [25] that $\dot{p}(t)^{\top}+p(t)^{\top} B_{1}^{*}(t) \leq-p(t)^{\top} / 2$ for all $t \geq 0$. Hence, Assumptions 4-5 hold with $\mathfrak{c}_{1}=0.5, \mathfrak{c}_{2}=e^{-1 / 4}$, $\mathfrak{c}_{3}=2 e^{1 / 4}, \mathfrak{c}_{5}=2 \sqrt{e}$, and $\mathfrak{c}_{6}=3 / 2$. The desired exponential stability result follows.

Remark 2. Under our assumption $\tau \in(0,1 /(3+2 \sqrt{e}))$, we can also make the nonlinear tracking dynamics for (36) and $(\sin (t), 0,0)^{\top}$ globally (instead of just locally) asymptotically stable. In fact, we can take $v_{1}(t)=\cos (t+\tau)-a_{0} \arctan \left(\gamma_{1}(t)\right)$ and $v_{2}(t-\tau)=-\gamma_{2}(t-\tau)-\left[\mathcal{G}(t) v_{1}(t-\tau) \xi_{2}(t-\tau)-\right.$ $\left.\xi_{3}(t-\tau)\left\{\sin (t+\tau)-a_{0}^{2} \arctan \left(\gamma_{1}(t-2 \tau)\right) /\left(1+\gamma_{1}^{2}(t-\tau)\right)\right\}\right]$, where $\gamma_{1}(t)=\xi_{1}(t)-\sin (t), \gamma_{2}(t)=\xi_{2}(t)+\mathcal{G}(t) \xi_{3}(t-\tau)$,
$\mathcal{G}(t)=\cos (t+\tau)-a_{0} \arctan \left(\gamma_{1}(t-\tau)\right)$, and $a_{0}$ is any constant such that

$$
a_{0} \in\left(0, \frac{1}{4 \pi}\right) \text { and } \frac{8-17 \pi a_{0}}{6\left(2+\pi a_{0}\right)^{2}}>\tau .
$$

See [26] for the proof that the preceding controls render the tracking dynamics GAS to 0 .

\section{CONCLUSIONS}

Proving stability for time-varying linear and neutral systems with delays is a challenging problem that is beyond the scope of standard methods. However, such systems are ubiquitous in engineering applications. While powerful, prediction methods are largely limited to building delay compensating controllers, so prediction may not always be applicable when the delays are not in the input. While there is a sizable literature on stabilizing linear time-varying systems under delays, the existing results usually require constructing functionals that may not always be so easy to find [13]. Here we presented new stability analysis techniques for time delay systems, including neutral systems where there is a delay in the time derivative of the state. Our main tools were linear Lyapunov functionals, and nonnegative or positive systems. However, no nonnegativity or positivity conditions are required for the given original systems. We hope to broaden our results to cover state dependent delays [31,32] and discrete-time analogs. We also hope to merge our results with the adaptive control work [33] on robustness under state constraints.

\section{ACKNOWLEDGEMENT}

The work of the second author was sponsored in part by NSF/EPAS Grant 1102348.

\section{REFERENCES}

[1] Bekiaris-Liberis, N., and Krstic, M., 2013. Nonlinear Control Under Nonconstant Delays. Advances in Design and Control Series. Society for Industrial and Applied Mathematics, Philadelphia, PA.

[2] Bekiaris-Liberis, N., and Krstic, M., 2013. "Robustness of nonlinear predictor feedback laws to time- and state-dependent delay perturbations". Automatica, 49(6), pp. 1576-1590.

[3] Germani, A., Manes, C., and Pepe, P., 2003. "Input-output linearization with delay cancellation for nonlinear delay systems: the problem of the internal stability". International Journal of Robust and Nonlinear Control, 13(9), pp. 909-937.

[4] Bonnet, C., Fioravanti, A., and Partington, J., 2011. "Stability of neutral systems with commensurate delays and poles asymptotic to the imaginary axis". SIAM Journal on Control and Optimization, 49(2), pp. 498-516. 
[5] Mazenc, F., and Ito, H., 2013. "Lyapunov technique and backstepping for nonlinear neutral systems". IEEE Transactions on Automatic Control, 58(2), pp. 512-517.

[6] Brayton, R., 1967. "Nonlinear oscillations in a distributed network". Quarterly of Applied Mathematics, 24, pp. 289301.

[7] Karimi, H., 2010. "Further results on stability analysis of time-delayed systems with nonlinear uncertainties". Inernational Journal of Systems and Control, 1-2010(2), pp. 56-61.

[8] Kolmanovskii, V., and Myshkis, A., 1999. Introduction to the Theory and Applications of Functional Differential Equations. Kluwer, Dordrecht, The Netherlands.

[9] Hale, J., and Verduyn Lunel, S., 1993. Introduction to Functional Differential Equations. Applied Mathematical Sciences Volume 99. Springer, New York.

[10] Haddad, W., Chellaboina, V., and Hui, Q., 2010. Nonnegative and Compartmental Dynamical Systems. Princeton University Press, Princeton, NJ.

[11] Haddad, W., and Chellaboina, V., 2004. "Stability and dissipativity theory for nonnegative and compartmental dynamical systems with time delay". Systems and Control Letters, 51(5), pp. 355-361.

[12] Krstic, M., 2009. Delay Compensation for Nonlinear, Adaptive, and PDE Systems. Systems and Control: Foundations and Applications. Birkhauser, Boston, MA.

[13] Pepe, P., Karafyllis, I., and Jiang, Z.-P., 2008. "On the Liapunov-Krasovskii methodology for the ISS of systems described by coupled delay differential and difference equation". Automatica, 49(9), pp. 2266-2273.

[14] Mazenc, F., Niculescu, S.-I., and Krstic, M., 2012. "Lyapunov-Krasovskii functionals and application to input delay compensation for linear time-invariant systems". $\mathrm{Au}$ tomatica, 48(7), pp. 1317-1323.

[15] Karafyllis, I., Pepe, P., and Jiang, Z.-P., 2009. "Stability results for systems described by coupled retarded functional differential equations and functional difference equations". Nonlinear Analysis: Theory, Methods and Applications, 71(7-8), pp. 3339-3362.

[16] Mazenc, F., accepted, 2014. "Stability analysis of timevarying neutral time-delay systems". IEEE Transactions on Automatic Control.

[17] Efimov, D., Perruquetti, W., and Richard, J.-P., 2013. "On reduced-order interval observers for time-delay systems". In Proceedings of the European Control Conference, pp. 2116-2121.

[18] Mazenc, F., Malisoff, M., and Niculescu, S.-I., 2014. "Reduction model approach for linear time-varying systems with delay". IEEE Transactions on Automatic Control, 59(8), pp. 2068-2082.

[19] Mazenc, F., and Niculescu, S.-I., 2011. "Generating positive and stable solutions through delayed state feedback".
Automatica, 47(3), pp. 525-533.

[20] Karafyllis, I., and Krstic, M., 2012. "Nonlinear stabilization under sampled and delayed measurements, and with inputs subject to delay and zero-order hold". IEEE Transactions on Automatic Control, 57(5), pp. 1141-1154.

[21] Efimov, D., Perruquetti, W., Raissi, T., and Zolghadri, A., 2013. "Interval observers for time-varying discretetime systems". IEEE Transactions on Automatic Control, 58(12), pp. 3218-3224.

[22] Mazenc, F., Dinh, T., and Niculescu, S.-I., to appear, 2014. "Interval observers for discrete-time systems". International Journal of Robust and Nonlinear Control.

[23] Germani, A., Manes, C., and Palumbo, P., 2010. "Representation of a class of MIMO systems via internally positive realization". European Journal of Control, 16(3), pp. 291-304.

[24] Cacace, F., Germani, A., Manes, C., and Setola, R., 2012. "A new approach to the internal positive representation of linear MIMO systems". IEEE Transactions on Automatic Control, 57(1), pp. 119-134.

[25] Mazenc, F., and Malisoff, M., to appear, 2014. "New technique for stability analysis for time-varying systems with delay". In Proceedings of the 53rd IEEE Conference on Decision and Control.

[26] Mazenc, F., and Malisoff, M., submitted, 2014. "Stabilizing time-varying systems with delay using linear Lyapunov functions and a positive systems approach".

[27] Mazenc, F., and Bernard, O., 2010. "Asymptotically stable interval observers for planar systems with complex poles". IEEE Transactions on Automatic Control, 55(2), pp. 523527.

[28] Mazenc, F., and Bernard, O., 2011. "Interval observers for linear time-invariant systems with disturbances". Automatica, 47(1), pp. 140-147.

[29] Malisoff, M., and Mazenc, F., 2009. Constructions of Strict Lyapunov Functions. Communications and Control Engineering Series. Springer, New York.

[30] Mazenc, F., and Malisoff, M., 2013. "Asymptotic stabilization for feedforward systems with delayed feedback". Automatica, 49(3), pp. 780-787.

[31] Bekiaris-Liberis, N., and Krstic, M., 2012. "Compensation of state-dependent input delay for nonlinear systems". IEEE Transactions on Automatic Control, 58(2), pp. 275289.

[32] Bekiaris-Liberis, N., Jankovic, M., and Krstic, M., 2012. "Compensation of state-dependent state delay for nonlinear systems". Systems and Control Letters, 61(8), pp. 894856.

[33] Malisoff, M., and Zhang, F., 2013. "Adaptive control for planar curve tracking under controller uncertainty". Automatica, 49(5), pp. 1411-1418. 